\title{
COMMENTARY
}

\section{Risk stratification in critically ill patients: GDF-15 scores in adult respiratory distress syndrome}

\author{
Tibor Kempf* and Kai C Wollert \\ See related by Clark et al., http://ccforum.com/content/17/3/R92
}

\begin{abstract}
Patients with adult respiratory distress syndrome (ARDS) are highly heterogeneous but current therapies are rather uniform and largely supportive. In the previous issue of Critical Care, Clark and colleagues report that the biomarker GDF-15 provides prognostic information in ARDS that is additive to that provided by the APACHE III score. Patients with high levels of growth-differentiation factor 15 (GDF-15) had a higher mortality and more complicated hospital course. Biomarkers such as GDF-15 may help us to identify patients at higher risk who may eventually benefit from more personalized and targeted therapies.
\end{abstract}

Adult respiratory distress syndrome (ARDS) is an acute inflammatory condition characterized by diffuse alveolar damage leading to pulmonary dysfunction and impaired survival. Owing to improved therapeutic strategies, such as lung protective ventilation and conservative fluid management, mortality in ARDS has declined but still remains unacceptably high. Mortality in ARDS is determined by several interrelated factors, including the severity of the underlying disease, comorbidities, respiratory failure, and the development of multi-organ failure. Thus, patients with ARDS are highly heterogeneous, and survival may vary considerably between patient subgroups.

In this context, Clark and colleagues [1] have determined the prognostic implications of the plasma biomarker growth-differentiation factor 15 (GDF-15) in a large cohort of 400 patients with ARDS previously enrolled in the Fluid and Catheter Treatment trial [1]. GDF-15 levels were highly elevated in ARDS and almost all patients had GDF-15 levels above 1,200 ng/L, the upper limit of normal. Higher circulating levels of

*Correspondence: kempf.tibor@mh-hannover.de

Klinik für Kardiologie und Angiologie, Medizinische Hochschule Hannover, Carl-Neuberg-Straße 1, 30625 Hannover, Germany
GDF-15 were associated with an increased risk of death and a more complicated hospital course during the first 60 days. Mortality was extremely high at $49 \%$ in the top quartile of GDF-15 (above 10,150 ng/L) compared to only $12 \%$ in the bottom quartile (below 3,585 ng/L). Patients in the top quartile of GDF-15 had only 2 ventilator free days and not a single ICU free day, whereas patients from the bottom quartile had 22 ventilator free days and 20 ICU free days. Similarly, GDF-15 was associated with the number of days with multi-organ failure. Importantly, these associations persisted after adjustment for disease severity using the APACHE III score, which reflects pulmonary, cardiovascular, central nervous system, renal, and hepatic disease dimensions.

Clark and colleagues confirmed the APACHE III score as a valuable but imperfect tool for risk stratification in ARDS as reflected by its area under the receiver operating characteristic curve (AUC) of 0.72 for the prediction of 60 day mortality (a perfect tool would have an AUC of 1.0). Notably, a single measurement of GDF-15 also had an AUC of 0.72, and combination of the APACHE III score with GDF-15 achieved an AUC of 0.77, which represents a considerable improvement in discriminatory precision [2].

GDF- 15 is a member of the TGF- $\beta$ cytokine family that is expressed in pathological conditions associated with acute or chronic tissue injury. On a molecular level, expression of GDF-15 is controlled by the ubiquitous transcription factor p53, which responds to stressors such as hypoxia, ischemia, oxidative stress, and inflammation. The circulating levels of GDF-15 are thought to reflect these stressors and their impact on disease progression and prognosis. As a result, GDF-15 has emerged as a powerful prognostic marker in several acute conditions such as acute myocardial infarction, pulmonary embolism, or stroke [3-5], and chronic disease states, including coronary artery disease, heart failure, idiopathic pulmonary arterial hypertension, and chronic kidney disease [6-8]. Thus, the very high levels of GDF-15 in ARDS may be related to the disease process itself and its impact on end-organ oxygenation and function, and to several pre-existing co-morbidities. 
The interesting study from Clark and colleagues will surely stimulate further research that should examine, for example, if serial measurements of GDF-15 are useful for monitoring response to therapy in ARDS. A recent study in patients with terminal heart failure showed that even very high levels of GDF-15 are remarkably dynamic and can be reversed by a potentially life-saving therapy (implantation of a left ventricular assist device in the case of heart failure) [9]. Considering the complex and multifactorial etiology of ARDS, and the association of GDF-15 to acute tissue injury and chronic disease burden, it will be interesting to explore if GDF-15 can also predict the development of ARDS in patients admitted to an ICU. Moreover, as discussed by Clark and colleagues, an independent prognostic marker such as GDF-15 may eventually prove useful in identifying high risk individuals for more aggressive or more specific therapies. Despite the highly heterogeneous patient population presenting with ARDS, current therapies are rather uniform and largely supportive. We must do better, and biomarkers such as GDF-15 may help us to develop more personalized and targeted therapies for this deadly condition.

\section{Abbreviations}

ARDS, adult respiratory distress syndrome; AUC, area under the receiver operating characteristic curve; GDF-15, growth-differentiation factor 15.

\section{Competing interests}

Drs Kempf and Wollert are named as co-inventors on a patent for the use of GDF-15 for cardiovascular applications and have a contract with Roche Diagnostics for the development of a GDF-15 assay.

\section{Author details}

Department of Cardiology and Angiology, Hannover Medical School, Hannover, Germany

Published: 31 July 2013

\section{References}

1. Clark BJ, Bull TM, Benson AB, Stream AR, Macht M, Gaydos J, Meadows C, Burnham EL, Moss M: Growth differentiation factor-15 and prognosis in acute respiratory distress syndrome: a retrospective cohort study. Crit Care 2013, 17:R92.

2. Pencina MJ, D'Agostino RB, Pencina KM, Janssens AC, Greenland P: Interpreting incremental value of markers added to risk prediction models. Am J Epidemiol 2012, 176:473-481.

3. Wollert KC, Kempf T, Peter T, Olofsson S, James S, Johnston N, Lindahl B, HornWichmann R, Brabant G, Simoons ML, Armstrong PW, Califf RM, Drexler H, Wallentin L: Prognostic value of growth-differentiation factor-15 in patients with non-ST-elevation acute coronary syndrome. Circulation 2007, 115:962-971.

4. Lankeit M, Kempf T, Dellas C, Cuny M, Tapken H, Peter T, Olschewski M, Konstantinides S, Wollert KC: Growth differentiation factor-15 for prognostic assessment of patients with acute pulmonary embolism. Am 」 Respir Crit Care Med 2008, 177:1018-1025.

5. Worthmann H, KempfT, Widera C, Tryc AB, Goldbecker A, Ma YT, Deb M, Tountopoulou A, Lambrecht J, Heeren M, Lichtinghagen R, Wollert KC, Weissenborn K: Growth differentiation factor 15 plasma levels and outcome after ischemic stroke. Cerebrovasc Dis 2011, 32:72-78.

6. Nickel N, Kempf T, Tapken H, Tongers J, Laenger F, Lehmann U, Golpon H, Olsson K, Wilkins MR, Gibbs JS, Hoeper MM, Wollert KC: Growth differentiation factor-15 in idiopathic pulmonary arterial hypertension. Am J Respir Crit Care Med 2008, 178:534-541.

7. KempfT, Sinning JM, Quint A, Bickel C, Sinning C, Wild PS, Schnabel R, Lubos E, Rupprecht HJ, Münzel T, Drexler H, Blankenberg S, Wollert KC: Growthdifferentiation factor- 15 for risk stratification in patients with stable and unstable coronary heart disease: results from the AtheroGene study. Circ Cardiovasc Genet 2009, 2:286-292.

8. Anand IS, Kempf T, Rector TS, Tapken H, AllhoffT, Jantzen F, Kuskowski M, Cohn JN, Drexler H, Wollert KC: Serial measurement of growthdifferentiation factor-15 in heart failure: relation to disease severity and prognosis in the Valsartan Heart Failure Trial. Circulation 2010, 122:1387-1395.

9. Lok SI, Winkens B, Goldschmeding R, van Geffen AJ, Nous FM, van Kuik J, van der Weide P, Klöpping C, Kirkels JH, Lahpor JR, Doevendans PA, de Jonge N, de Weger RA: Circulating growth differentiation factor-15 correlates with myocardial fibrosis in patients with non-ischaemic dilated cardiomyopathy and decreases rapidly after left ventricular assist device support. Eur J Heart Fail 2012, 14:1249-1256.

doi:10.1186/cc12765

Cite this article as: Kempf T, Wollert KC: Risk stratification in critically ill patients: GDF-15 scores in adult respiratory distress syndrome. Critical Care 2013, 17:173. 